\title{
Flattening filter-free technique in volumetric modulated arc therapy for lung stereotactic body radiotherapy: A clinical comparison with the flattening filter technique
}

\author{
SHURI AOKI, HIDEOMI YAMASHITA, AKIHIRO HAGA, KANABU NAWA, TOSHIKAZU IMAE, \\ WATARU TAKAHASHI, OSAMU ABE and KEIICHI NAKAGAWA
}

Department of Radiology, University of Tokyo Hospital, Tokyo 113-8655, Japan

Received September 9, 2017; Accepted December 13, 2017

DOI: $10.3892 / 01.2018 .7809$

\begin{abstract}
The present study sought to evaluate the impact of the flattening filter-free (FFF) technique in volumetric modulated arc therapy for lung stereotactic body radiotherapy. Its clinical safety and availability were compared with the flattening filter (FF) method. The cases of 65 patients who underwent lung volumetric modulated arc therapy-stereotactic body radiotherapy (VMAT-SBRT) using FF or FFF techniques were reviewed. A total of $55 \mathrm{~Gy} / 4$ fractions (fr) was prescribed for peripheral lesions or $56 \mathrm{~Gy} / 7 \mathrm{fr}$ for central lesions. The total monitor units (MU), treatment time, dose to tumors, dose to organs at risk, tumor control (local control rate, overall survival, progression-free survival) and adverse events between cases treated with FF and cases treated with the FFF technique were compared. A total of 35 patients were treated with conventional FF techniques prior to November 2014 and 30 patients were treated with FFF techniques after this date. It was revealed that the beam-on time was significantly shortened by the FFF technique $(\mathrm{P}<0.01)$. Other factors were
\end{abstract}

Correspondence to: Dr Hideomi Yamashita or Dr Shuri Aoki, Department of Radiology, University of Tokyo Hospital, 3-7-1 Hongo, Bunkyo-ku, Tokyo 113-8655, Japan

E-mail: yamachan07291973@yahoo.co.jp

E-mail: daisyshuri@yahoo.co.jp

Abbreviations: AE, adverse event; $\mathrm{BED}$, biologically equivalent dose; CT, computed tomography; DVH, dose volume histogram; FF, flattening filter; FFF, flattening filter-free; GGO, ground glass opacity; GTV, gross tumor volume; HI, homogeneity index; ITV, internal target volume; LCR, local control rate; MLC, multileaf collimator; MU, monitor unit; NSCLC, non-small cell lung cancer; OARs, organs at risk; PFS, progression-free survival; PTV, planning target volume; RFS, relapse-free survival; SBRT, stereotactic body radiotherapy; TPS, treatment planning system; TT, treatment time; VMAT, volumetric modulated arc therapy

Key words: flattening filter-free, stereotactic body radiotherapy, volumetric modulated arc therapy, lung cancer, radiation pneumonitis similar for FFF and FF plans in respect to conformity $(\mathrm{P}=0.95)$, homogeneity $(\mathrm{P}=0.20)$ and other dosimetric values, including total MU and planning target volume/internal target volume coverage. The median follow-up period was 18 months (range, 2-35). One-year local control rates were 97.1 and $90.0 \%$ in the FF group and FFF groups, respectively $(\mathrm{P}=0.33)$. Grade 3 pneumonitis was observed in 5.8\% of FF patients and 3.4\% of FFF patients $(\mathrm{P}=1.00)$. No other adverse events $\geq$ grade 3 were observed. The results of the study suggest that VMAT-SBRT using the FFF technique shortens the treatment time for lung SBRT while maintaining a high local control rate with low toxicity.

\section{Introduction}

For early-stage non-small cell lung cancer (NSCLC), surgery is still the first choice for treatment $(1,2)$. However, not all early-stage NSCLC patients are suited for surgery because of advanced age, patient refusal, or other reasons $(3,4)$. Stereotactic body radiotherapy (SBRT) has been widely used as an effective alternative treatment for primary lung tumors, and reports excellent 3-year progression-free survival (PFS) of up to $90 \%$, which is considerably better than past data with conventional radiotherapy $(5,6)$. In addition, several studies on SBRT in patients deemed operable have shown that SBRT is also an option for these patients (5-7). Furthermore, with the development of anticancer drugs and immunotherapy, long-term survival has been obtained even in cases with distant metastases. As several papers report the usefulness of SBRT for 'oligo-metastasis or oligo-recurrence,' SBRT is expected to play a role as radical local therapy for pulmonary oligo-recurrence (8-12).

In SBRT, good local control is obtained by increasing the dose per fraction, which extends the irradiation time per fraction; It makes the treatment stressful for elderly patients suffering from cardiopulmonary comorbidities, and ultimately increase the risk of intra-fraction motion (13-15). We have performed SBRT using a kind of intensity modulated radiation therapy (IMRT) method called single-arc volumetric modulated arc therapy (VMAT) created by SmartArc (Pinnacle; Philips Medical Systems B.V., Eindhoven, The Netherlands). VMAT allows reduction of SBRT treatment times for each 
fraction, but is still limited by the maximum dose rate of a conventional linear accelerator (linac) with a flattened beam (16).

Expecting to further shorten treatment time, we adopted the flattening filter-free (FFF) technique to our VMAT-SBRT system in 2014. Compensation of the forward-peak bremsstrahlung by a flattening-filter decreases the maximal dose output of a linac, increasing the treatment time (17). By removing the flattening filter (so called flattening-filter-free), it is possible to increase the dose rate and dramatically shorten the treatment time. Although the FFF technique may improve clinical safety and efficacy by reducing treatment time, few clinical data to support this are available. We evaluated the safety and availability of VMAT-SBRT using the FFF technique in treatment of primary and metastatic lung tumors.

\section{Patients and methods}

Patients and tumor characteristics. From November 2013 to October 2015, 77 consecutive patients with 79 pulmonary lesions received single-arc VMAT-SBRT at the University of Tokyo. Of 77 cases, 10 were excluded due to lack of imaging examination after treatment, and the remaining 67 cases were included. Cases of obvious idiopathic pulmonary fibrosis were excluded. Of these 67 patients, 35 patients (35 cases) were treated between November 2013 and October 2014 with the flattening filter (FF) technique, and 30 patients (32 lesions) between November 2014 and October 2015 were treated with the FFF technique. Among 54 primary lung tumors, tissue diagnosis had been obtained in 23 patients (43\%), including 5 squamous cell carcinomas and 18 adenocarcinomas. Stage classification of primary cases (8th edition of the UICC TNM classification) was $\leq 1 \mathrm{~A} 1,11 ; 1 \mathrm{~A} 2,22 ; 1 \mathrm{Ac}, 19 ; 2 \mathrm{~A}, 3$; $2 \mathrm{~B}, 3 ; \geq 3,4$. On computerized tomography (CT), a total of $85 \%$ of the primary tumors measured $\leq 3 \mathrm{~cm}$. A total of 54 cases $(83 \%)$ were solid tumors, 4 cases $(6 \%)$ were pure ground glass opacity (GGO), and $11(\%)$ were mixed. The study was approved by the ethics committee of University of Tokyo Hospital (3372-(3)/2016) and written informed consent was obtained from all patients.

Treatment planning. Planned dose was 55 Gy in four fractions (biologically effective dose using the LQ model with a The alpha/beta=10 Gy: $\mathrm{BED}_{10}=105.8 \mathrm{~Gy}$ ) for 55 peripheral lesions, or $56 \mathrm{~Gy}$ in seven fractions $\left(\mathrm{BED}_{10}=100.8 \mathrm{~Gy}\right)$ for 14 central lesions to cover $95 \%$ of the PTV $\left(\mathrm{D}_{95 \%}\right)$. Central tumors were defined as such using RTOG 0236 criteria $(18,19)$.

All patients underwent CT based SBRT planning for VMAT. Four-dimensional (4D) CT images for treatment planning were acquired with 2 -mm-thick sections using a 16-detector scanner (Aquilion LB ${ }^{\circledR}$; Toshiba Medical Systems, Otawara, Japan). The patients were treated in the supine position while wearing an abdominal compressor. A stereotactic body frame was also used to minimize breathing artifacts for treatment planning CT. Scans were performed using an external respiratory monitoring system (AZ-733 V ${ }^{\circledR}$; Anzai Medical, Tokyo, Japan). In our institution, 4D-CT for planning divides the respiratory cycle into 10 sections. Respiratory phase data were transferred to a treatment planning system (TPS) (Pinnacle ${ }^{3 \circledast}$, version 9.10; Philips Medical Systems B.V.). Gross tumor volume (GTV) was delineated in each respiratory phase using the lung window (window, 1,600 HU; level, -300 HU). These 10 GTVs were fused to form the internal target volume (ITV) and then a uniform $5 \mathrm{~mm}$ margin was added to create the planning target volume (PTV) (20-22).

Dose to targets and OARs. Planning target coverage aimed to cover PTV with $95 \%$ of the prescribed dose. The main organs at risk (OARs) were healthy lung, spinal cord, heart, and esophagus. Treatment plans were required to meet explicit objectives as follows: V20 $<10 \%$ (less than $10 \%$ of the volume receiving $20 \mathrm{~Gy}$ ) and V5 $<25 \%$ for the ipsilateral lung, V20 $<0 \%$ and V5 $<15 \%$ for the contralateral lung, V15=0\% for spinal cord, $\mathrm{V} 30=0 \%$ for heart and liver, and $\mathrm{V} 50=0 \%$ for body (23).

SBRT procedure. All patients were treated using VMAT-SBRT with 6 or $10 \mathrm{MV}$ FF or FFF beams. The maximum dose rate for FFF beams was 1500 monitor units (MU)/min for $6 \mathrm{MV}$ and 2,400 MU/min for $10 \mathrm{MV}$ (18). VMAT plans were designed using a single partial arc with angle ranges of $-40^{\circ}$ to $180^{\circ}$ (left lung) or $-180^{\circ}$ to $40^{\circ}$ (right lung) (Fig. 1).

A conformal field shape was used to reduce the interplay effect (24). In inverse planning of VMAT, the conformal-like field shape can be created by imposing a constraint on multi-leaf collimator (MLC) motion speed (25); the MLC moves smoothly during VMAT delivery, so that the constraint is intended to form field shapes that do not hide the target. In this study, a constraint on MLC motion of $0.2 \mathrm{~cm} /$ degree was applied.

Dosimetric planning and plan analysis were generated with the TPS. The collapsed cone convolution method (which is comparable to the superposition method) in the TPS was used for heterogeneity correction for the lungs. All final calculations were performed with a grid size of $2.0 \mathrm{~mm}$. Dose distributions were calculated using peak exhalation CT data.

Evaluation of dosimetric and technical data. For each group of patients, technical parameters of dose delivery were scored in terms of total number of MU, MU/Gy, and beam-on times. Dosimetric quality of treatments was measured from dose volume histogram (DVH) analysis. For the PTV and ITV, the target coverage (mean, $\mathrm{D}_{2 \%}, \mathrm{D}_{50 \%}, \mathrm{D}_{98 \%}$ ), homogeneity, and conformity were reported. The homogeneity index (HI) was described as $\left(\mathrm{D}_{2 \%}-\mathrm{D}_{98 \%}\right) / \mathrm{D}_{50 \%}$, and the conformity index as the ratio of the volume receiving $95 \%$ of the prescribed dose and the PTV/ITV volume $(19,26)$. For OARs, the mean dose, maximum dose (Dmax) and appropriate volumes of $\mathrm{Vx}$ (volume receiving at least X Gy) were scored.

Evaluation of clinical data. The patients were usually examined two months after SBRT and every six months thereafter. Chest $\mathrm{CT}$ or ${ }^{18} \mathrm{~F}$-fluorodeoxyglucose positron emission tomography/computed tomography (FDG-PET/CT) was used for follow-up. Local tumor recurrences or distant relapses were evaluated following the Response Evaluation Criteria In Solid Tumors (RECIST) criteria (version 1.1). In case RECIST criteria were not useful depending on the post-irradiation changes of normal tissue, an FDG-PET/CT was performed to differentiate between tumor recurrence and lung fibrosis. 


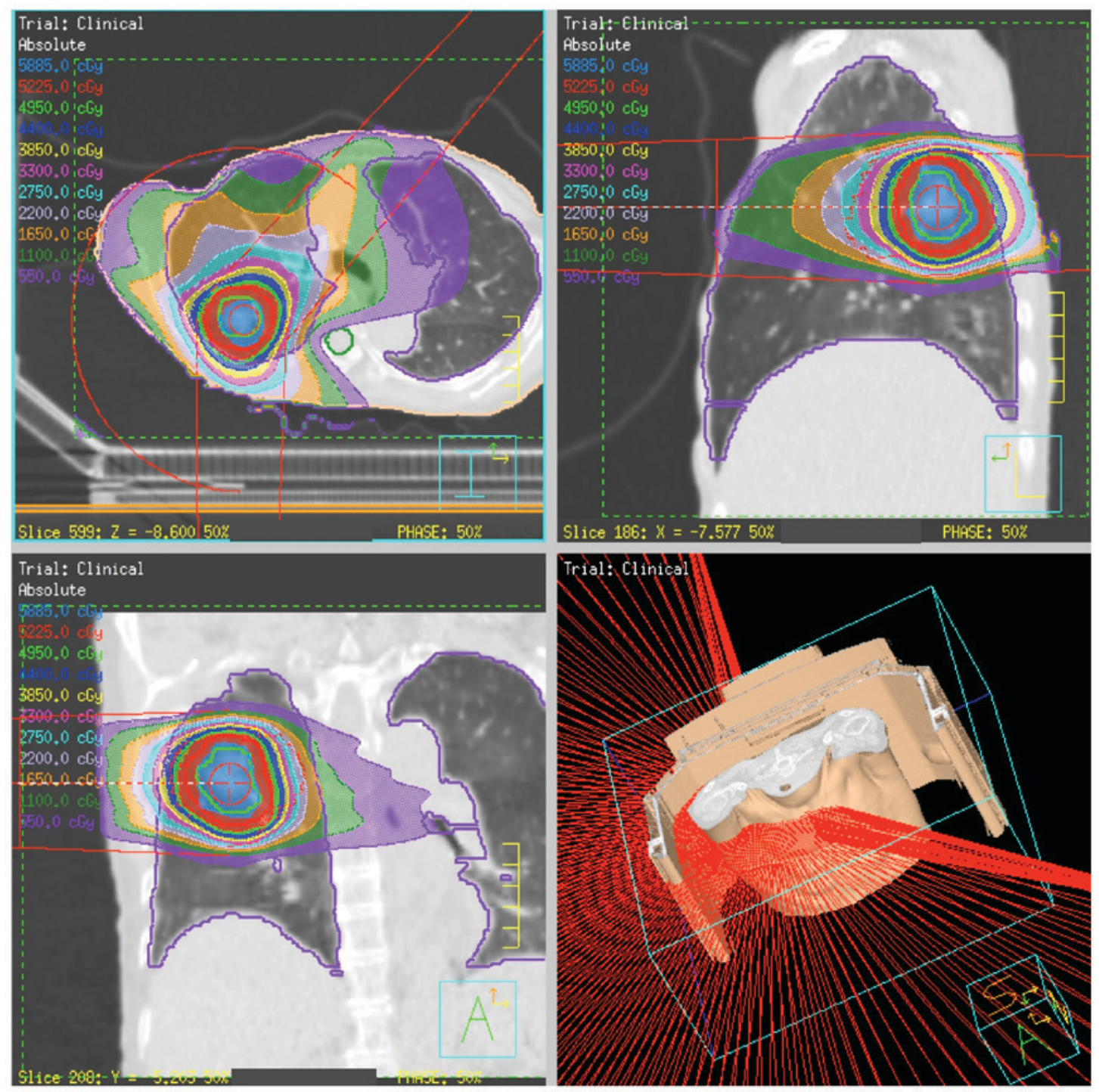

Figure 1. Radiation dose distribution of SBRT using intensity modulated radiation therapy method called single-arc volumetric modulated arc therapy.

The 1-year local control rate (LCR), overall survival (OS), and relapse-free survival (RFS) were evaluated and compared between FF and FFF cases. Acute and late toxicities were assessed according to the Common Terminology Criteria for Adverse Events Version 4.0 (CTCAE v4.0).

Statistical analysis. The OS, LCR and RFS were defined over the period from the time of the first day of SBRT until death, recurrence or the last patient contact. Local failure was defined as progressive and increasing CT scan abnormalities with a high maximum 2-(fluorine-18)-fluoro-2-deoxy-D-glucose (FDG) uptake of $>3$ standardized uptake value (SUV), with or without biopsy. They were calculated using Kaplan-Meier curves, and the curves were then compared using the log-rank test. The statistical analyses were performed using R software, and $\mathrm{P}<0.05$ was considered to indicate a statistically significant difference.

\section{Results}

Characteristics of each treatment group. Table I compares the characteristics of the FF and FFF groups. There was no significant difference in the age distribution of subjects in the groups, and $>50 \%$ patients in both groups were $>80$ years old. The mean PTV of the FF and FFF groups was $50.4 \mathrm{~cm}^{3}$ (range, 14.1-225.63) and $40.1 \mathrm{~cm}^{3}$ (range, 9.0-135.2), ITV volume was $17.1 \mathrm{~cm}^{3}$ (range, 2.3-103.3) and $14.8 \mathrm{~cm}^{3}$ (range, 1.4-67.2), respectively. There were no significant differences between PTV and ITV ( $\mathrm{P}=0.32,0.64)$, respectively. Regarding tumor location, in the second half period using FFF, the proportion of central lesions had a tendency being larger (11 to $31 \%, \mathrm{P}=0.069$ ). Of the 35 patients in the FF group, 30 of 35 lesions ( $86 \%$, including 25 primary cases) were NSCLC, while in the FFF group, 30 lesions (94\%, including 29 primary cases) in 32 patients were NSCLC, the rest being pulmonary oligo-recurrence.

Table II summarizes the tumor features, focusing on primary NSCLC in both groups. The pathological diagnosis of NSCLC was confirmed in 42\%, of which adenocarcinoma: squamous cell carcinoma ratios were 8:2 and 9:3, respectively. In the FF and FFF groups, solid lesions accounted for 76 and $81 \%$ of each, respectively.

There was no significant difference in the distribution of tumor sizes (solid part) between the groups $(\mathrm{P}=0.15)$. Tumors 
Table I. Patient background and comparison between the FF and FFF groups.

\begin{tabular}{|c|c|c|c|c|c|}
\hline & $\begin{array}{c}\text { Total } \\
\text { mean (range) }\end{array}$ & $\mathrm{FF}$ & FFF & P-value & Test \\
\hline \multicolumn{6}{|l|}{ Number } \\
\hline Patients-lesions & $65-67$ & $35-35$ & $30-32$ & & \\
\hline \multicolumn{6}{|l|}{ Sex } \\
\hline Male & 40 & 22 & 18 & 0.81 & $\chi^{2}$ \\
\hline Female & 25 & 13 & 12 & & \\
\hline Mean-age (range) & & $77(56-89)$ & $76(46-86)$ & 0.85 & Unpaired $t$ \\
\hline \multicolumn{6}{|l|}{ Age-classification } \\
\hline$\geqq 80$ years & 32 & 17 & 15 & 0.91 & $\chi^{2}$ \\
\hline$<80$ years & 33 & 18 & 15 & & \\
\hline \multicolumn{6}{|l|}{ General condition } \\
\hline Karnofsky index & & $90(70-100)$ & $90(80-100)$ & 0.69 & Unpaired $t$ \\
\hline \multicolumn{6}{|l|}{ Smoking status } \\
\hline Active & 6 & 3 & 3 & & \\
\hline Former & 22 & 10 & 12 & & \\
\hline Never & 23 & 10 & 13 & & \\
\hline Not known & 14 & 12 & 2 & & \\
\hline Brickman index & & $450(0-2,000)$ & $630(0-3,000)$ & 1 & Unpaired $t$ \\
\hline \multicolumn{6}{|l|}{ Pulmonary lesions } \\
\hline Primary NSCLC & 54 & 25 & 29 & & \\
\hline Recurrent/residual NSCLC & 6 & 5 & 1 & & \\
\hline Metastatic pulmonary lesions & 7 & 5 & 2 & & \\
\hline \multicolumn{6}{|l|}{ Tumor location } \\
\hline Peripheral & 51 & 33 & 22 & 0.05 & $\chi^{2}$ \\
\hline Central & 14 & 4 & 10 & & \\
\hline \multicolumn{6}{|l|}{ Involved lobe } \\
\hline Right upper & 20 & 10 & 10 & & \\
\hline Right middle/lower & 21 & 13 & 8 & & \\
\hline Left upper & 8 & 5 & 3 & & \\
\hline Left lower & 15 & 9 & 6 & & \\
\hline Other & 5 & 0 & 5 & & \\
\hline \multicolumn{6}{|l|}{ PTV } \\
\hline $\mathrm{cm}^{3}$ & $45.6(9.0-225.6)$ & $50.4(14.1-225.6)$ & $40.1(9.0-135.2)$ & 0.32 & Unpaired $\mathrm{t}$ \\
\hline \multicolumn{6}{|l|}{ ITV } \\
\hline $\mathrm{cm}^{3}$ & $16.0(1.4-103.3)$ & $17.1(2.3-103.3)$ & $14.8(1.4-67.2)$ & 0.64 & Unpaired $\mathrm{t}$ \\
\hline
\end{tabular}

FF, flattening filter; FFF, flattening filter free; PTV, planning treatment volume; ITV, internal target volume; U, upper; M, middle; L, lower.

were classified based on the T classification of the UICC eighth edition as shown in Table II.

Dosimetric and technical data. Technical features for the FF and FFF groups are summarized in Table III. Between FF and FFF, no statistical difference was observed for PTV/ITV coverage in terms of $\mathrm{D}_{98 \%}$ and $\mathrm{D}_{50 \%}$. There was no significant difference between the two groups even at $\mathrm{D}_{2 \%}$, rather as an indicator of HI. The FFF plans were not inferior, with a mean conformity index of 1.32 (range 1.04-3.11), while the FF plans showed 1.51 (range 0.82-2.21) for PTV $(\mathrm{P}=0.95)$. The same was true for ITV. The difference in the HI of the two techniques was also not significant $(\mathrm{P}=0.20)$. Comparing the mean $\mathrm{MU}$ values, no significant differences were found between the two groups too $(\mathrm{P}=0.63)$.

On the other hand, the beam-on time was significantly shortened with use of the FFF plan $(\mathrm{P}<0.01)$ (Fig. 2); the mean beam-on-time of the FFF plan was less than half that of the FF plan. This is in agreement with other studies (27-29).

Dose to the OARs. Table IV shows the doses to the OARs with the FF and the FFF techniques. No significant difference was observed between the two treatment plans for the lung dose, in terms of the mean dose (D mean) or the V20, V10, or V5. We 
Table II. The tumor features, focusing on primary NSCLC in both groups.

\begin{tabular}{lcccc}
\hline Primary NSCLC & FF & FFF & P-value & Test \\
\hline Number of lesions & 25 & 27 & & \\
Pathology & & & & \\
Adenocarcinoma & 8 & 9 & 0.91 & $\chi^{2}$ \\
Squamous cell & 2 & 3 & & \\
Others & 0 & 0 & & \\
Unknown & 15 & 15 & & \\
Lesion texture & & & & \\
GGO & 3 & 1 & 0.53 & $\chi^{2}$ \\
Part-solid & 3 & 4 & & \\
Solid & 19 & 22 & & \\
Tumor diameter & & & & \\
Mean (range) & 22.9 & 24.0 & 0.68 & Unpaired t \\
& $(0-51)$ & $(4-60)$ & & \\
T-Stage & & & & \\
T0 & 3 & 1 & 0.65 & $\chi^{2}$ \\
T1A1 & 1 & 1 & & \\
T1A2 & 12 & 12 & & \\
T1A3 & 6 & 8 & & \\
T1B & 1 & 1 & & \\
T2A & 1 & 2 & & \\
T2B & 1 & 2 & & \\
\end{tabular}

NSCLC, non-small cell lung carcinoma; GGO, ground glass opacity.

evaluated the dose to other OARS, and found no significant differences between the FF and the FFF plans.

Tumor control. The median follow-up period was 18 months (range 2-35) for all, and 24 months for the FF group (range 2-35) and 12 months for the FFF group (range 2-23). The median OS, PFS and LCR times had not been reached at the time of data analysis, and no significant differences were observed ( $\mathrm{P}=0.164,0.26,0.847$, respectively). Kaplan-Meier analyses of OS and RFS in both groups are shown in Figs. 3 and 4, respectively. Fig. 5 is a Kaplan-Meier curve for local control. One-year LCRs were $97.1 \%$ (1 case relapsed) and $90.0 \%$ ( 3 cases relapsed) in the FF and FFF groups, respectively.

Out of 67 subjects, recurrence was observed in 10 cases ( 7 cases in the FF group and 3 cases in the FFF group). There were 6 cases of local recurrence and 10 cases of distant metastasis. All cases with local recurrence also had distant metastases. One case of recurrence is shown in Fig. 6.

Adverse events. We investigated the frequency of adverse events (AEs) based on the information in the medical records using CTCAE v4.0. Toxicity observed within 90 days of SBRT was categorized as acute, whereas toxicity observed $>90$ days after SBRT was categorized as late.

The appearance of AEs in each group is shown in Table V. Acute adverse events of grade $\geq 3$ were not observed. There were six cases $(9.5 \%)$ of dermatitis, seven cases $(11.1 \%)$ of pleurisy
Table III. Technical features for FF and FFF techniques.

\begin{tabular}{cccc}
\hline & FF mean (range) & FFF mean (range) & P-value \\
\hline PTV & & & \\
$\mathrm{D}_{2 \%}$ & $61.9(59.4-76.4)$ & $62.5(52.7-69.6)$ & 0.72 \\
$\mathrm{D}_{98 \%}$ & $54.1(50.4-67.5)$ & $54.2(49.0-54.9)$ & 0.85 \\
$\mathrm{D}_{50 \%}$ & $59.0(50.4-67.5)$ & $59.1(51.6-63.3)$ & 0.81 \\
$\mathrm{CI}$ & $1.51(0.82-2.21)$ & $1.32(1.04-3.11)$ & 0.95 \\
$\mathrm{HI}$ & $0.15(0.04-0.36)$ & $0.13(0.06-0.26)$ & 0.20 \\
$\mathrm{ITV}$ & & & \\
$\mathrm{D}_{2 \%}$ & $62.7(59.6-75.3)$ & $63.2(52.7-70.4)$ & 0.71 \\
$\mathrm{D}_{98 \%}$ & $57.5(54.0-61.0)$ & $57.6(51.3-59.8)$ & 0.94 \\
$\mathrm{D}_{50 \%}$ & $60.6(57.6-69.7)$ & $60.6(51.9-64.2)$ & 0.52 \\
$\mathrm{CI}$ & $1.51(0.82-2.21)$ & $1.32(1.04-3.11)$ & 0.95 \\
$\mathrm{HI}$ & $0.15(0.04-0.36)$ & $0.13(0.06-0.26)$ & 0.20 \\
MU & $2,181(1,203.5-2,414.2)$ & $2,076(718.5-2,506.0)$ & 0.63 \\
$\mathrm{TT}$ & $3.64(2.00-4.02)$ & $1.48(0.50-1.80)$ & 0.010 \\
$(\mathrm{~min})$ & & & \\
\hline
\end{tabular}

PTV, planning treatment volume; ITV, internal target volume; $\mathrm{D}_{2 \%}\left({ }_{98 \% / 50 \%}\right)$, dose at which the volume irradiated above the dose is $2 \%$ of the total volume of the organ of interest; MU, monitor unit; TT, treatment time; CI, conformity index; HI, homogeneity index; FF, flattening filter; FFF, flattening filter-free. All P-values were calculated using unpaired t test.

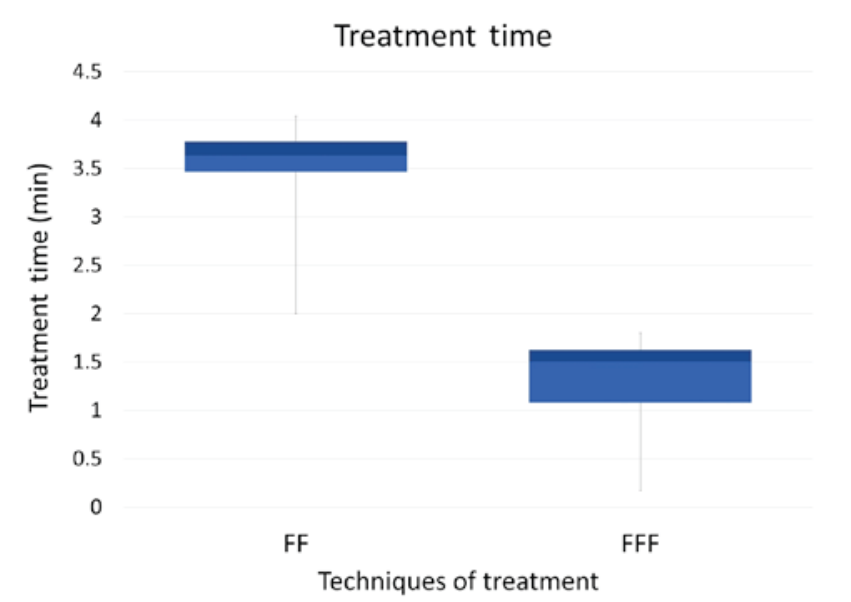

Figure 2. Box-plot diagrams about the treatment time of the FF and FFF groups.

and two cases (3.2\%) of esophagitis among all subjects. Late adverse events were investigated in 63 cases (FF: 34 cases, FFF: 29 cases) that could be observed for $>90$ days from the start of treatment. In these 63 cases, grade $\geq 1$ radiation-induced pneumonitis were observed in 33 cases (97\%) in the FF group and 27 cases (93\%) in the FFF group. Most of the 'pneumonitis' showed only changes on the image, in other words, grade 1. Of the FF group, there were twenty-nine cases (85\%) of grade1 pneumonitis, three cases (9\%) of grade 2, and two cases (6\%) of grade 3. Of the FFF group, there were twenty-two cases (76\%) of grade1 pneumonitis, four cases (14\%) of grade 2 and one case $(4 \%)$ of grade 3 . Pneumonitis of grade $\geq 4$ was not observed. 
Table IV. Summary of dose volume histogram analysis for organs at risk.

\begin{tabular}{llccr}
\hline Organ & Dose volume & FF $(\mathrm{n}=35)$ mean (range) & FFF (n=30) mean (range) & P-value \\
\hline Lung & V20 Gy (\%) & $8.0(1.8-16.9)$ & $7.9(2.7-15.4)$ & 0.90 \\
& V10 Gy (\%) & $14.2(2.5-36.7)$ & $14.5(6.4-29.0)$ & 0.82 \\
& V5 Gy (\%) & $21.6(4.9-49.2)$ & $21.8(5.2-42.6)$ & 0.94 \\
Spinal cord & Dmean (cGy) & $5.50(0.2-11.5)$ & $5.5(2.0-9.6)$ & 0.99 \\
Heart & Dmax (cGy) & $13.8(4.2-27.8)$ & $11.60(0.2-25.7)$ & 0.16 \\
\hline
\end{tabular}

FF, flattening filter; FFF, flattening filter-free; V20 (V10, V5), percentage volume receiving 20 Gy (10 Gy, 5 Gy); Dmean, the mean dose. All P-values were calculated using unpaired $t$ test.

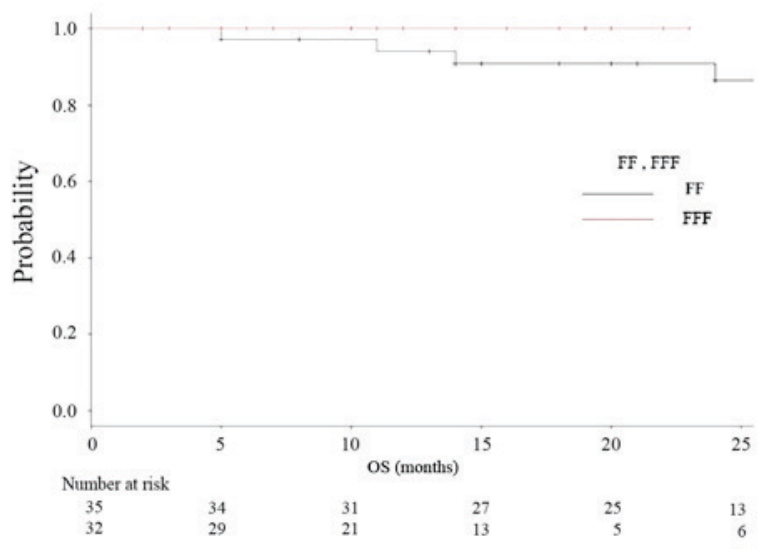

Figure 3. Kaplan-Meier curves illustrating overall survival for patients treated using FF technique (black line) and FFF technique (red line).

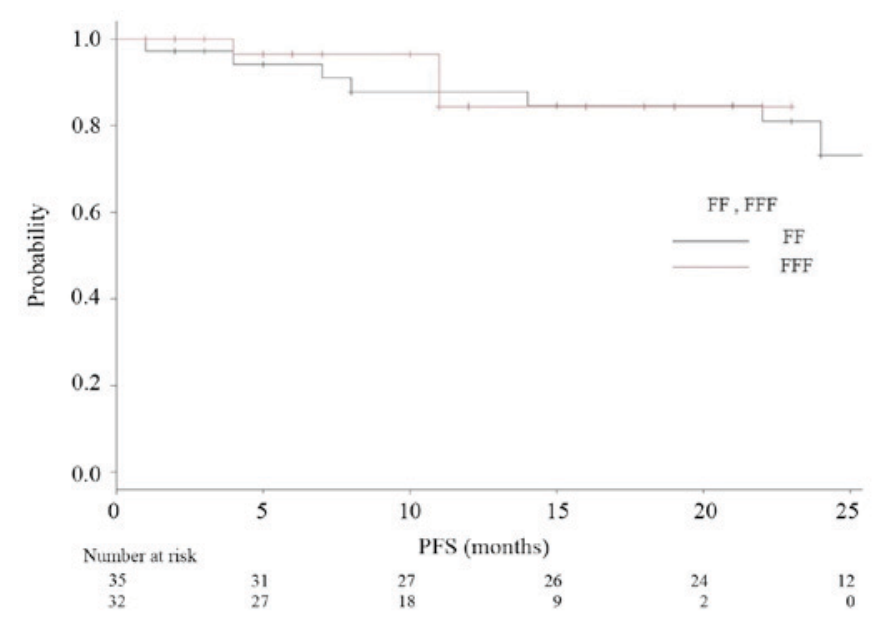

Figure 4. Kaplan-Meier curves for relapse-free survival (RFS) for patients treated using FF technique (black line) and FFF technique (red line).

We experienced one case of grade 2 tracheal stenosis in the FFF group. In this case, wheezing appeared 3 months after treatment, and the patient received steroid medication. At 7 months after treatment, thickening of the bronchial walls and tracheal stenosis were observed, but symptoms were controlled by the use of inhaled steroids (Fig. 7). There were no late adverse events of grade $\geq 3$ involving the heart or esophagus.

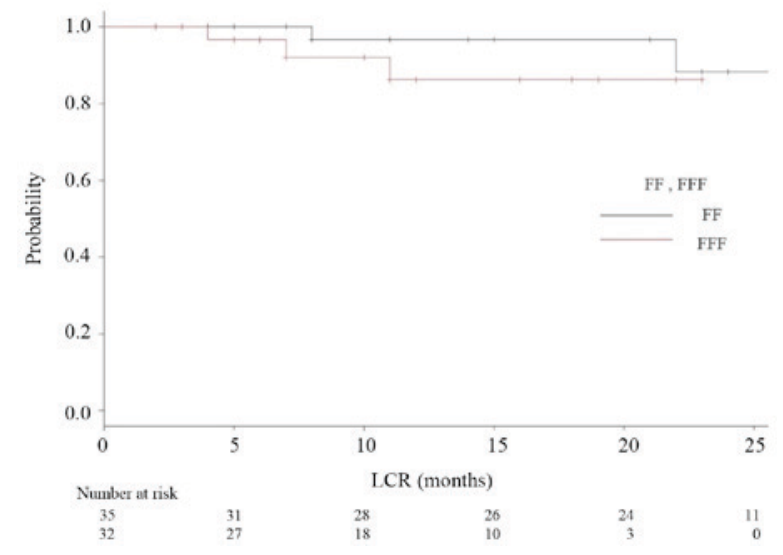

Figure 5. Kaplan-Meier curves for local control rate (LCR) for patients treated using FF technique (black line) and FFF technique (red line).
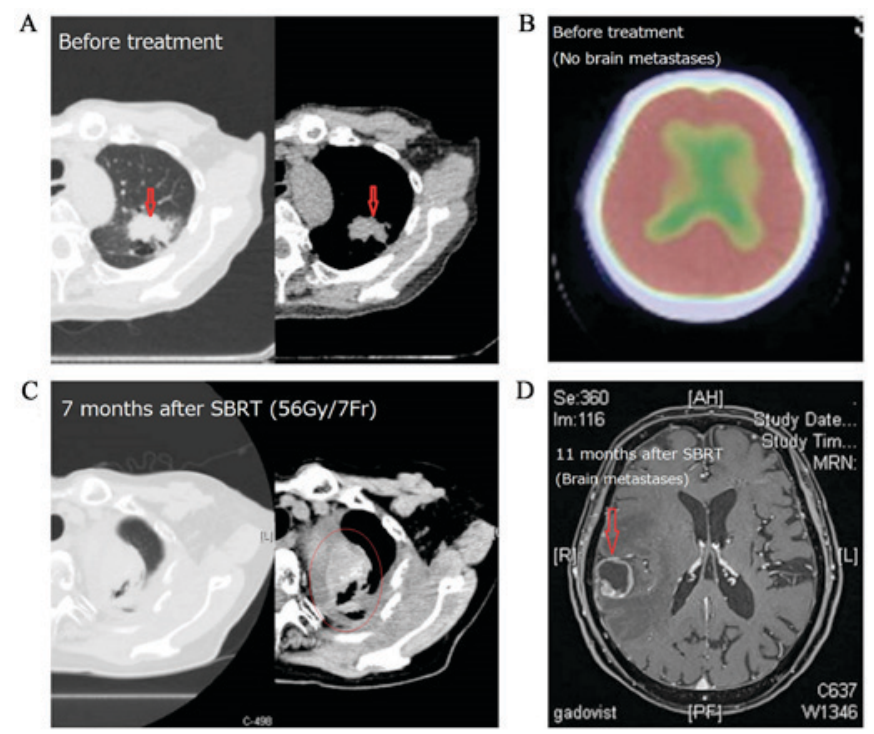

Figure 6. In this case of primary lung cancer located in the left upper lobe, local recurrence occurred 7 months and brain metastasis occurred 11 months after SBRT (55 Gy/4Fr) was performed. (A) Primary lung lesion before treatment, (B) PET-CT image of brain before SBRT, (C) local recurrence 7 months after SBRT, (D) brain metastasis 11 months after SBRT.

\section{Discussion}

Clinical reports on FFF are sparse, and few of these compare 
Table V. The appearance of side effects in each group.

\begin{tabular}{lccc}
\hline $\begin{array}{l}\text { Component } \\
\text { and disorders }\end{array}$ & $\begin{array}{c}\text { Grade } \\
\text { (CTCAE vol4.0.) }\end{array}$ & $\begin{array}{c}\text { FF-group } \\
\text { number }(\%)\end{array}$ & $\begin{array}{c}\text { FFF-group } \\
\text { number }(\%)\end{array}$ \\
\hline $\begin{array}{l}\text { Acute adverse } \\
\text { events }\end{array}$ & 35 & 30 \\
Dermatitis & $\leq 2$ & $5(14.3)$ & $1(3.3)$ \\
Esophagitis & 3 & $0(0)$ & $0(0)$ \\
& $\leq 2$ & $2(5.7)$ & $0(0)$ \\
Late adverse & 3 & $0(0)$ & $0(0)$ \\
events & & 34 & 29 \\
Pneumonitis & 1 & $28(82.4)$ & $3(10.3)$ \\
& 2 & $3(8.8)$ & $1(4.3)$ \\
Tracheal stenosis & 3 & $2(5.9)$ & $1(4.3)$ \\
& $\leq 2$ & $0(0)$ & $0(0)$ \\
Pleuritis & 3 & $0(0)$ & $2(8.7)$ \\
Pericarditis & $\leq 2$ & $5(14.7)$ & $0(0)$ \\
Rib fracture & 3 & $0(0)$ & $1(4.3)$ \\
& $\leq 2$ & $0(0)$ & $0(0)$ \\
& 3 & $0(0)$ & $0(0)$ \\
& all & $0(0)$ & $0(0)$
\end{tabular}

FF, flattening filter; FFF, flattening filter free; CTCAE, common terminology criteria for adverse events.

the clinical results of FF and FFF in the same facility. The present study is a retrospective observation study of 67 cases where VMAT-SBRT was performed in our hospital from 2013 to 2015. The cases during the period were divided into FF and FFF groups, and beam data and clinical results were compared between the two groups. Although the two groups underwent treatment at different times in the same facility, no change was made except for the presence or absence of the flattening filter.

Shortening of treatment time. We have performed SBRT using VMAT-IMRT since 2010, and FFF since 2014. Yamashita, et al already reported details of treatment from our hospital (20). VMAT has been reported to be a novel rotational technique and an extension of IMRT, and is applicable for SBRT for lung tumors (23,30-34). By introducing VMAT, we could reduce the dose delivery time down to $210 \mathrm{sec}$ for a D95 prescription dose of $50 \mathrm{~Gy}$ in four fractions (35). Furthermore, we added a FFF-system to our LINAC (Elekta AB, Stockholm, Sweden), and succeeded further reduction of treatment time with the beam-on time reduced $50 \%$ or more (22) (Table III, Fig. 2).

Clinical Merit of FFF system. Increasing the dose rate and shortening the treatment time reduces the patient's pain. As Thomas et al stated, shortening treatment time enables more accurate dose delivery because body motion and irregular breathing during treatment time are reduced (36).

Another advantage of the FFF method is reported to be a reduction in the dose to surrounding normal tissue, in particular lungs, by drawing sharp slope on the periphery

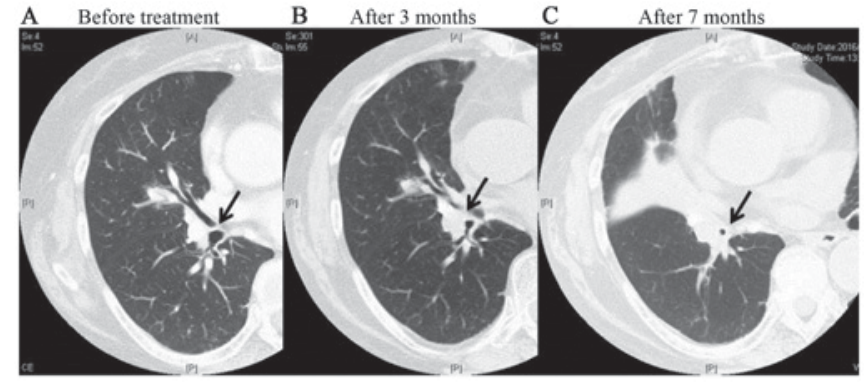

Figure 7. A case of grade 2 bronchial stenosis in an FFF subject. This case received VMAT-SBRT of $56 \mathrm{~Gy} / 7 \mathrm{fr}$ using FFF technique for central lesions in the right lung. It is obvious that the bronchial lumen indicated by the arrow gradually narrowed after treatment. (A) Before treatment; (B) 3 months after treatment; and (C) 7 months after treatment.

of the tumor $(37,38)$. It is expected to lead to reduce side effects eventually. In addition, adopting FFF methodology to volume prescription, a high dose (so-called 'hot spot') will be aimed at the central part of the target. This may improve the treatment of radio-resistant lesions (e.g., colon cancer lung oligo-recurrence) (39).

These advantages of using FFF-method were not directly reflected in our clinical data, however. There was no significant difference between the FF and FFF groups in MU value, dose to OARs and targets. It seems to be partly because the number of the subjects of this study was small, the stage and tumor diameters had a wide range, and the observation period was short. Prendergast et al reported that there was no significant difference between the two treatments, and Rieber et al pointed out the same cause as us $(40,41)$.

As for the clinical result in our research, one-year LCR was 95\% for all, $93 \%$ for the FF group and $97 \%$ for the FFF group. One-year OS was $97 \%$ for all, $94 \%$ for FF and $100 \%$ for FFF group, respectively. Rieber, et al reported the results similar to ours. They achieved 1-year LCR of $92.8 \%$ and 1-year OS of $94.4 \%$ with FFF-VMAT-SBRT using 8x7.5 Gy $\left(\mathrm{D}_{80 \%}\right)$ for central lesions and $3 \times 15$ Gy $\left(D_{65 \%}\right)$ for peripheral lesions (14). Navarria et al reported on 132 VMAT-SBRT cases with $48 \mathrm{~Gy} / 4$ fr prescriptions with a 1 year LCR of $100 \%$ with FFF and $92.5 \%$ with FF (27). In their report, they stated that the FFF achieved a significantly better one-year LCR. However, most of the presentations so far are only saying that there is no obvious difference for the same reasons as ours $(14,28,29)$. These results can lead to no definite conclusion at the moment, but at least, we can say that the performance of FFF was not inferior to that of FF.

Speaking of AEs of this study using FFF, grade 3 AEs appeared as two cases of pneumonitis $(3 \%)$ and one case of esophagitis (1.5\%). No AEs > grade 3 were observed. Although the observation period was short and no conclusions can be drawn, no serious AEs have been observed in either group to date. Although most reports targeted peripheral early stage lung cancer, the target of our study was not limited to early stage, and also included central lesions that are said to have higher risk of AEs $(5,42,43)$. In addition, most studies of lung SBRT in Japan adopt a 48 Gy/4 fr prescription $(5,44)$, whereas we prescribed $55 \mathrm{~Gy} / 4$ fr for peripheral lung cancers and $56 \mathrm{~Gy} / 7 \mathrm{fr}$ for central ones (that is, $\mathrm{BED}_{10}>100$ in all cases). 
Taking these conditions into consideration, safety results were not inferior to those of other reports about SBRT using FF technique.

Limitations of this study are its small number of cases and short observation period. Further, the study was conducted at a single facility and the observation period between the two groups varied. It is inevitable for retrospective observational studies to have missing data, such as loss of pathological diagnosis. Although the results of the SBRT of each facility cannot be compared simply because the techniques, prescription dose, and objects are greatly different from each other, it will be necessary to conduct investigations with larger numbers of cases and longer observation periods.

VMAT-SBRT using FFF enabled a shortened treatment time without lowering local control or increasing AEs.

\section{Acknowledgements}

This study was partially supported by a Grant-in-Aid from JSPS (Japan Society for the Promotion of Science) KAKENHI JP Scientific Research (C), grant no. 15K08692.

\section{References}

1. Maquilan $G$ and Timmerman R: Stereotactic body radiation therapy for early-stage lung cancer. Cancer $\mathbf{J} 22$ : 274-279, 2016.

2. Ginsberg RJ and Rubinstein LV: Randomized trial of lobectomy versus limited resection for T1 N0 non-small cell lung cancer. Lung cancer study group. Ann Thorac Surg 60: 615-623, 1995.

3. Falkson CB, Vella ET, Yu E, El-Mallah M, Mackenzie R, Ellis PM and Ung YC: Radiotherapy with curative intent in patients with early-stage, medically inoperable, non-small-cell lung cancer: A systematic review. Clin Lung Cancer 18: 105-121.e5, 2017.

4. Nanda RH, Liu Y, Gillespie TW, Mikell JL, Ramalingam SS, Fernandez FG, Curran WJ, Lipscomb J and Higgins KA: Stereotactic body radiation therapy versus no treatment for early stage non-small cell lung cancer in medically inoperable elderly patients: A National Cancer Data Base analysis. Cancer 121: 4222-4230, 2015.

5. Nagata Y, Hiraoka M, Shibata T, Onishi H, Kokubo M, Karasawa K, Shioyama Y, Onimaru R, Kozuka T, Kunieda E, et al: Prospective trial of stereotactic body radiation therapy for both operable and inoperable T1N0M0 non-small cell lung cancer: Japan Clinical Oncology Group Study JCOG0403. Int J Radiat Oncol Biol Phys 93: 989-996, 2015.

6. Chang JY, Senan S, Paul MA, Mehran RJ, Louie AV, Balter P, Groen HJ, McRae SE, Widder J, Feng L, et al: Stereotactic ablative radiotherapy versus lobectomy for operable stage I non-small-cell lung cancer: A pooled analysis of two randomised trials. Lancet Oncol 16: 630-637, 2015.

7. Baumann P, Nyman J, Hoyer M, Wennberg B, Gagliardi G, Lax I, Drugge N, Ekberg L, Friesland S, Johansson KA, et al: Outcome in a prospective phase II trial of medically inoperable stage I non-small-cell lung cancer patients treated with stereotactic body radiotherapy. J Clin Oncol 27: 3290-3296, 2009.

8. Niibe Y and Hayakawa K: Oligometastases and oligo-recurrence: The new era of cancer therapy. Jpn J Clin Oncol 40: 107-111, 2010.

9. Niibe Y and Chang JY: Novel insights of oligometastases and oligo-recurrence and review of the literature. Pulm Med 2012: 261096, 2012.

10. Yamashita H, Niibe Y, Yamamoto T, Katsui K, Jingu K, Kanazawa S, Terahara A and Nakagawa K: Lung stereotactic radiotherapy for oligometastases: Comparison of oligo-recurrence and sync-oligometastases. Jpn J Clin Oncol 46: 687-691, 2016.

11. Niibe Y, Yamashita H, Sekiguchi K, Takahashi W, Shiraishi K, Okuma K, Terahara A, Kawamori J and Nakagawa K: Stereotactic body radiotherapy results for pulmonary oligometastases: A two-institution collaborative investigation. Anticancer Res 35: 4903-4908, 2015.
12. Pasqualetti F, Montrone S, Vivaldi C, Zani M, Fedele D, Fornaro L, Pasqualetti G, Salvatore L, Manfredi B, Laliscia C, et al: Stereotactic body radiotherapy in patients with lung oligometastases from colorectal cancer. Anticancer Res 37: 315-319, 2017.

13. Zhang JY, Lu JY, Wu LL, Hong DL, Ma CC, Peng X and Lin ZX: A dosimetric and treatment efficiency evaluation of stereotactic body radiation therapy for peripheral lung cancer using flattening filter free beams. Oncotarget 7: 73792-73799, 2016.

14. Rieber J, Tonndorf-Martini E, Schramm O, Rhein B, König L, Adeberg S, Meyerhof E, Mohr A, Kappes J, Hoffmann H, et al: Establishing stereotactic body radiotherapy with flattening filter free techniques in the treatment of pulmonary lesions-initial experiences from a single institution. Radiat Oncol 11: 80, 2016.

15. Hoogeman MS, Nuyttens JJ, Levendag PC and Heijmen BJ: Time dependence of intrafraction patient motion assessed by repeat stereoscopic imaging. Int J Radiat Oncol Biol Phys 70: 609-618, 2008.

16. Takahashi W, Yamashita H, Kida S, Masutani Y, Sakumi A, Ohtomo K, Nakagawa K and Haga A: Verification of planning target volume settings in volumetric modulated arc therapy for stereotactic body radiation therapy by using in-treatment 4-dimensional cone beam computed tomography. Int J Radiat Oncol Biol Phys 86: 426-431, 2013.

17. Stieler F, Fleckenstein J, Simeonova A, Wenz F and Lohr F: Intensity modulated radiosurgery of brain metastases with flattening filter-free beams. Radiother Oncol 109: 448-451, 2013.

18. Timmerman RD, Paulus R, Galvin J, Michalski J, Straube W, Bradley J, Fakiris A, Bezjak A, Videtic G, Johnstone D, et al: Stereotactic body radiation therapy for medically inoperable early stage lung cancer. JAMA 303: 1070-1076, 2010.

19. Roesch J, Panje C, Sterzing F, Mantel F, Nestle U, Andratschke N and Guckenberger M: SBRT for centrally localized NSCLC-What is too central? Radiat Oncol 11: 157, 2016.

20. Yamashita H, Takahashi W, Haga A, Kida S, Saotome N and Nakagawa K: Stereotactic body radiotherapy for small lung tumors in the University of Tokyo hospital. Biomed Res Int 2014: 136513,2014

21. Nakagawa K, Haga A, Kida S, Masutani Y, Yamashita H, Takahashi W, Sakumi A, Saotome N, Shiraki T, Ohtomo K, et al: $4 \mathrm{D}$ registration and $4 \mathrm{D}$ verification of lung tumor position for stereotactic volumetric modulated arc therapy using respiratory-correlated cone-beam CT. J Radiat Res 54: 152-156, 2013.

22. Nakagawa K, Haga A, Sakumi A, Yamashita H, Igaki H, Shiraki T, Ohtomo K, Iwai Y and Yoda K: Impact of flattening-filter-free techniques on delivery time for lung stereotactic volumetric modulated arc therapy and image quality of concurrent kilovoltage cone-beam computed tomography: A preliminary phantom study. J Radiat Res 55: 200-202, 2014.

23. Yamashita H, Haga A, Takahashi W, Takenaka R, Imae T, Takenaka $S$ and Nakagawa K: Volumetric modulated arc therapy for lung stereotactic radiation therapy can achieve high local control rates. Radiat Oncol 9: 243, 2014.

24. Dvorak P, Georg D, Bogner J, Kroupa B, Dieckmann K and Pötter R: Impact of IMRT and leaf width on stereotactic body radiotherapy of liver and lung lesions. Int J Radiat Oncol Biol Phys 61: 1572-1581, 2005.

25. Haga A, Kida S, Saotome N, Takahashi W, Yamashita H, Masutani Y et al: Others: Four-dimensional Cone-Beam CT During SBRT. In: Stereotactic Body Radiation Therapy. Springer, Tokyo, pp225-236 2015.

26. Miura H, Ozawa S, Hosono F, Sumida N, Okazue T, Yamada K and Nagata Y: Gafchromic EBT-XD film: Dosimetry characterization in high-dose, volumetric-modulated arc therapy. J Appl Clin Med Phys 17: 312-322, 2016.

27. Navarria P, Ascolese AM, Mancosu P, Alongi F, Clerici E, Tozzi A, Iftode C, Reggiori G, Tomatis S, Infante M, et al: Volumetric modulated arc therapy with flattening filter free (FFF) beams for stereotactic body radiation therapy (SBRT) in patients with medically inoperable early stage non small cell lung cancer (NSCLC). Radiother Oncol 107: 414-418, 2013.

28. Prendergast BM, Fiveash JB, Popple RA, Clark GM, Thomas EM, Minnich DJ, Jacob R, Spencer SA, Bonner JA and Dobelbower MC: Flattening filter-free linac improves treatment delivery efficiency in stereotactic body radiation therapy. J Appl Clin Med Phys 14: 4126, 2013.

29. Lang S, Shrestha B, Graydon S, Cavelaars F, Linsenmeier C, Hrbacek J, Klöck S, Studer G and Riesterer O: Clinical application of flattening filter free beams for extracranial stereotactic radiotherapy. Radiother Oncol 106: 255-259, 2013. 
30. Zhang GG, Ku L, Dilling TJ, Stevens CW, Zhang RR, Li W and Feygelman V: Volumetric modulated arc planning for lung stereotactic body radiotherapy using conventional and unflattened photon beams: A dosimetric comparison with 3D technique. Radiat Oncol 6: 152, 2011.

31. Macchia G, Deodato F, Cilla S, Cammelli S, Guido A, Ferioli M, Siepe G, Valentini V, Morganti AG and Ferrandina G: Volumetric modulated arc therapy for treatment of solid tumors: Current insights. Onco Targets Ther 10: 3755-3772, 2017.

32. Nakagawa K, Haga A, Shiraishi K, Yamashita H, Igaki H, Terahara A, Ohtomo K, Saegusa S, Shiraki T, Oritate T and Yoda K: First clinical cone-beam CT imaging during volumetric modulated arc therapy. Radiother Oncol 90: 422-423, 2009.

33. Holt A, van Vliet-Vroegindeweij C, Mans A, Belderbos JS and Damen EM: Volumetric-modulated arc therapy for stereotactic body radiotherapy of lung tumors: A comparison with intensity-modulated radiotherapy techniques. Int J Radiat Oncol Biol Phys 81: 1560-1567, 2011.

34. Deodato F, Cilla S, Macchia G, Caravatta L, Mignogna S, Massaccesi M, Picardi V, Digesu C, Sallustio G, Bonomo P, et al: Extracranial radiosurgery with volumetric modulated arc therapy: Feasibility evaluation of a phase I trial. Oncol Lett 5: 1889-1896, 2013

35. Nakagawa K, Kida S, Haga A, Masutani Y, Yamashita H, Onoe T, Imae T, Tanaka K, Ohtomo K and Yoda K: 4D digitally reconstructed radiography for verifying a lung tumor position during volumetric modulated arc therapy. J Radiat Res 53: 628-632, 2012.

36. Thomas EM, Popple RA, Prendergast BM, Clark GM, Dobelbower MC and Fiveash JB: Effects of flattening filter-free and volumetric-modulated arc therapy delivery on treatment efficiency 14: 4328, 2013.

37. Cashmore J: The characterization of unflattened photon beams from a 6 MV linear accelerator. Phys Med Biol 53: 1933-1946, 2008.

38. Vassiliev ON, Titt U, Pönisch F, Kry SF, Mohan R and Gillin MT: Dosimetric properties of photon beams from a flattening filter free clinical accelerator. Phys Med Biol 51: 1907-1917, 2006.
39. Kinj R, Bondiau PY, François E, Gérard JP, Naghavi AO, Leysalle A, Chamorey E, Evesque L, Padovani B, Ianessi A, et al: Radiosensitivity of colon and rectal lung oligometastasis treated with stereotactic ablative radiotherapy. Clin Colorectal Cancer 16: e211-e220, 2016.

40. Rieber J, Tonndorf-Martini E, Schramm O, Rhein B, König L, Adeberg S, Meyerhof E, Mohr A, Kappes J and Hoffmann H: Establishing stereotactic body radiotherapy with flattening filter free techniques in the treatment of pulmonary lesions-initial experiences from a single institution. Radiat Oncol. 11: 80, 2016.

41. Prendergast BM, Dobelbower MC, Bonner JA, Popple RA, Baden CJ, Minnich DJ, Cerfolio RJ, Spencer SA and Fiveash JB: Stereotactic body radiation therapy (SBRT) for lung malignancies: Preliminary toxicity results using a flattening filter-free linear accelerator operating at 2,400 monitor units per minute. Radiat Oncol 8: 273, 2013.

42. Timmerman R, McGarry R, Yiannoutsos C, Papiez L, Tudor K, DeLuca J, Ewing M, Abdulrahman R, DesRosiers C, Williams M and Fletcher J: Excessive toxicity when treating central tumors in a phase II study of stereotactic body radiation therapy for medically inoperable early-stage lung cancer. J Clin Oncol 24: 4833-4839, 2006.

43. Roesch J, Panje C, Sterzing F, Mantel F, Nestle U, Andratschke N and Guckenberger M: SBRT for centrally localized NSCLC-What is too central? Radiat Oncol 11: 157, 2016.

44. Kimura T, Nagata Y, Eba J, Ozawa S, Ishikura S, Shibata T, Ito Y, Hiraoka M and Nishimura Y; Radiation Oncology Study Group of the Japan Clinical Oncology Group: A randomized Phase III trial of comparing two dose-fractionations stereotactic body radiotherapy (SBRT) for medically inoperable Stage IA non-small cell lung cancer or small lung lesions clinically diagnosed as primary lung cancer: Japan Clinical Oncology Group Study JCOG1408 (J-SBRT trial). Jpn J Clin Oncol, 2017 (Epub ahead of print). 